\title{
Heterogenization of Indium for the Friedel-Craft Benzoylation of Toluene
}

\author{
Farook ADAM ${ }^{*}$, Kei Lin SEK \\ School of Chemical Sciences, Universiti Sains Malaysia, Penang 11800, Malaysia
}

\begin{abstract}
Indium incorporated silica samples with different indium contents were synthesized using a template free sol-gel method. The silica used was extracted from rice husk (RH) and the resulting catalyst was labeled as RH- $x \mathrm{In}(x=5 \%, 10 \%, 15 \%$, and $20 \%)$. From the $\mathrm{N}_{2}$ adsorption analysis the presence of type IV isotherm in RH-blank and RH-xIn indicated the mesoporous nature of the catalysts. In the XRD pattern, a broad band at ca. $2 \theta=25^{\circ}$ was observed for all the catalysts which showed them to be amorphous. TEM micrographs revealed that the material is composed of nanoparticles. Friedel-Craft benzoylation of toluene was carried out using the RH-xIn catalyst. The optimum conditions for the benzoylation of toluene were determined and at $373 \mathrm{~K}, 100 \%$ conversion and $89 \%$ selectivity for the para-product (i.e. 4-methylbenzophenone) were obtained.
\end{abstract}

Key words: rice husk; sol-gel; indium; Friedel-Craft benzoylation; Toluene

CLC number: O643 Document code: A

Received 30 June 2012. Accepted 28 August 2012.

*Corresponding author.Tel: +60-46533567; Fax:+60-46574854; E-mail:farook@usm.my,farook_dr@yahoo.com

This work was supported by Universiti Sains Malaysia RU research grant (1001/PKIMIA/814019 and 1001/PKIMIA/811092) and Universiti

Sains Malaysia Graduate Assistantship to KLS.

English edition available online at Elsevier ScienceDirect (http://www.sciencedirect.com/science/journal/18722067).

Generally, sodium silicate is obtained by smelting quartz sand and sodium carbonate at $1027 \mathrm{~K}$ which requires a large amount of energy [1] or it was synthesized from commercial starting materials such as tetraethoxysilane (TEOS) [2,3] or tetramethoxysilane (TMOS) $[5,6]$. These procedures are not only costly, but harmful as well. Therefore, there is a need to investigate new procedures to overcome these disadvantages.

Rice husk ( $\mathrm{RH})$ is an agricultural biomass from the rice milling industry. Every ton of rice produces 0.23 tons of $\mathrm{RH}$ and about 100 million tons of RH is produced annually. The disposal of RH by burning produces toxic gases and fine ash that pollute the environment and living organisms [7]. Therefore, conversion of RH into useful material is beneficial to the environment. The high content of silica in $\mathrm{RH}$ makes it a potential material to be used as a source of pure silica. The silica extracted from RH has a high specific surface area, high porosity and high thermal stability [1]. Many researchers have reported the use of RH as a raw material to prepare silica supported heterogeneous catalysts [8,9].

In most reported cases the $\mathrm{RH}$ is calcined to form rice husk ash (RHA) before using it as a support or as an adsorbent. Chiarakorn et al. [7] reported that pure silicon dioxide can be obtained from RH. Furthermore, the high temperatures used in the calcination process of $\mathrm{RH}$ to obtain RHA should be avoided to reduce the cost. An alternative route to obtain the silica in $\mathrm{RH}$ has been developed by Adam et al. [10]. A similar method to prepare silica from
RH was reported by Dimitriev et al. [11] using the sol-gel approach which produces various morphological silica materials such as bulks, fiber sheets, coating films, and particles at low temperature. The sol-gel method is advantageous as it offers control in the preparation of silica catalysts, it is also environment friendly, low cost, and results in highly pure materials.

The product of the Friedel-Crafts benzoylation of toluene especially the para-product, i.e., 4-methylbenzophenone (4-MBP) is useful in many applications. It is an intermediate in the synthesis of pharmaceutical, fine chemical, and petroleum products, and is used in the perfume industry and in the production of dielectric fluids $[12,13]$. Traditionally, these reactions were carried out homogeneously using Lewis acid catalysts such as $\mathrm{AlCl}_{3}, \mathrm{FeCl}_{3}, \mathrm{BF}_{3}$, and $\mathrm{ZnCl}_{2}$. However, these hazardous catalysts have many disadvantages in product separation and catalyst recovery, as they are highly toxic and corrosive, and lead to environmental pollution [14-16].

To overcome these problems, many type of heterogeneous catalysts have been investigated, for example, zeolites $[12,14,17], \mathrm{WO}_{x} / \mathrm{ZrO}_{2}$ solid acid catalysts [18], $\mathrm{ZrO}_{2} / \mathrm{Al}_{2} \mathrm{O}_{3}$ core nanocomposite catalysts [19], potassium iron zirconium phosphates (PIZP) [13], and Si-MCM-41 [20,21]. However, these catalysts are expensive and their preparation involves calcination at high temperature, and the yield is low or requires long reaction times. Therefore, improved methods are worth pursuing to overcome these problems. 
In this study, indium was selected as the metal to be incorporated into silica due to its specific properties with respect to air stability and low toxicity [22]. Indium is one of the post-transition metals. It has been reported that indium will give a high selectivity at low metal loading in catalytic reactions such as Fiedel-Craft alkylation, acylation, Wagner-Meerwein rearrangement, and Diels-Alder reactions [8,23-25]. Herein, we report the use of a simple template free sol-gel method to prepare mesoporous indium-silica composite materials and its use as a heterogeneous catalyst.

\section{Experimental}

RH was obtained from a local rice mill in Penang. Nitric acid (QReC, 65\%), sodium hydroxide (Systerm, 99\%), toluene (QRec, 99\%), benzoyl chloride (Fluka, 99\%), dodecane (Fluka, 99\%), and indium nitrate (Alfa Aesar, $99 \%$ ) were all of high grade and used as received.

\subsection{Extraction of silica}

RH (30.0 g) was stirred with $500 \mathrm{ml}$ of $1.0 \mathrm{~mol} / \mathrm{L}$ nitric acid for $24 \mathrm{~h}$ at room temperature. The acid treated $\mathrm{RH}$ was washed with distilled water until neutral $\mathrm{pH}$ was achieved, to ensure that all acid had been removed. The treated $\mathrm{RH}$ was dried at $383 \mathrm{~K}$ in an oven for $24 \mathrm{~h}$ and the mass was recorded. It was then treated with $500 \mathrm{ml}$ of $1.0 \mathrm{~mol} / \mathrm{L}$ sodium hydroxide for $24 \mathrm{~h}$ at room temperature. The mixture was filtered to obtain a dark brown solution of sodium silicate. The dry mass of the insoluble waste was recorded to calculate the mass of the extracted silica in $\mathrm{RH}$.

\subsection{Synthesis of RH-blank}

The sodium silicate was titrated directly with $3.0 \mathrm{~mol} / \mathrm{L}$ nitric acid until $\mathrm{pH}$ 9. The resulting brownish gel was aged for $48 \mathrm{~h}$ at room temperature. Then, the gel was filtered by suction filtration and washed with hot distilled water. The white precipitate was dried at $383 \mathrm{~K}$ in an oven for $24 \mathrm{~h}$ and ground into a fine powder to form RH-blank. The yield was $3.05 \mathrm{~g}$.

\subsection{Synthesis of RH- $x$ In}

The required mass of the indium nitrate was calculated and dissolved in $3.0 \mathrm{~mol} / \mathrm{L}$ of nitric acid. The sodium silicate solution was titrated with this mixture of nitric acid and indium nitrate to $\mathrm{pH}$ 9. A brownish gel was formed. This was aged for $48 \mathrm{~h}$ and then washed with hot distilled water until the precipitate become white. The white solid was dried at $383 \mathrm{~K}$ in an oven for $24 \mathrm{~h}$ and then ground into a fine powder. RH- $x$ In was synthesized with different indium metal loadings of $5 \%, 10 \%, 15 \%$, and $20 \%$ and labeled as RH-5In, RH-10In, RH-15In, and RH-20In, respectively.

\subsection{Characterization of RH-xIn}

The catalysts RH-xIn and RH-blank were characterized by Fourier transform infrared (FTIR) spectroscopy, scanning electron microscopy (SEM), energy dispersive X-ray (EDX) spectroscopy, transmission electron microscopy (TEM), nitrogen adsorption-desorption analysis (BET), atomic absorption spectrometry (AAS), and powder X-ray diffraction (XRD).

\subsection{Friedel-Craft benzoylation of toluene}

The liquid phase Friedel-Craft benzoylation of toluene was carried out in a two-necked round bottom flask equipped with a reflux condenser. The flask was immersed in an oil bath to maintain the temperature and the reaction was run under dry Ar gas to avoid the effects of moisture. These reactions were run under different conditions to determine the optimum condition for the reaction. The reaction mixture $(0.5 \mathrm{ml})$ was withdrawn periodically with a syringe containing cotton wool, and added to $1.0 \mu \mathrm{l}$ of dodecane as the internal standard. The resulting solution was analyzed by gas chromatography (Clarus 500, PerkinElmer). The products were identified by gas chromatography equipped with mass spectroscopy (GC-MS, Clarus 600, PerkinElmer).

\section{Results and discussion}

The results show that the catalyst mass yield varies with the percentage of indium incorporation. The catalyst mass yield increased almost linearly as the metal loading was increased, i.e. $4.63 \mathrm{~g}$ (RH-5In), $5.11 \mathrm{~g}$ (RH-10In), $6.10 \mathrm{~g}$ (RH-15In), and $7.43 \mathrm{~g}$ (RH-20In). RH-blank was also run as the control or blank to compare the changes of the catalyst before and after the indium metal was added. RH-blank gave the lowest mass with $3.05 \mathrm{~g}$.

\subsection{Characterization results of the catalyst}

\subsubsection{FTIR analysis}

Figure 1 shows the FTIR spectra for RH-blank and RH-In with different metal loadings. The transmission bands at $3451 \mathrm{~cm}^{-1}$ (RH-blank, RH-5In, and RH-10In) and at 3449 $\mathrm{cm}^{-1}$ (RH-15In, and RH-20In) is due to the overlapping of the stretching vibration of the $\mathrm{O}-\mathrm{H}$ bond of silica and water. The bending vibrations of $\mathrm{O}-\mathrm{H}$ bond in the water molecules were observed at ca. 1638 and $1640 \mathrm{~cm}^{-1}$ for all the cata- 


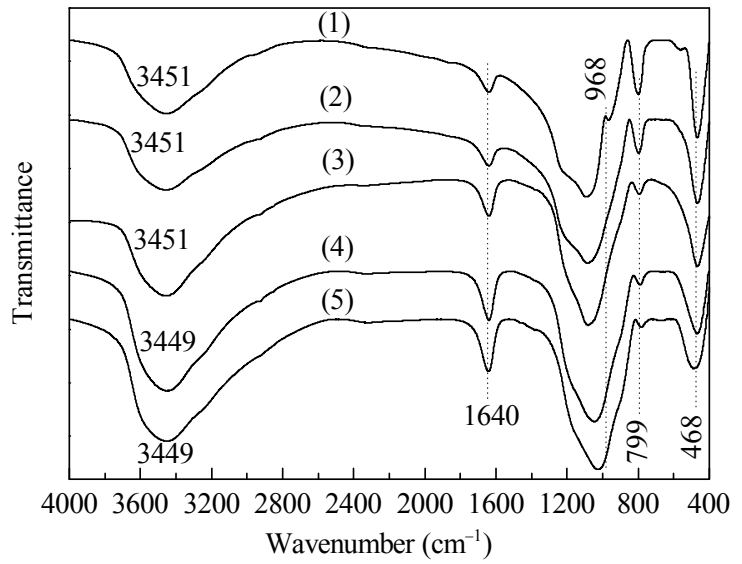

Fig. 1. FTIR spectra of RH-blank (1), RH-5In (2), RH-10In (3), RH-15In (4), and RH-20In (5).

lysts. The strong bands at 1080 and $1088 \mathrm{~cm}^{-1}$ were due to the symmetric vibration of siloxane, $\mathrm{Si}-\mathrm{O}-\mathrm{Si}$ bond. Both $\mathrm{Si}-\mathrm{O}-\mathrm{Si}$ stretching and bending vibrations were observed at ca. $780-799 \mathrm{~cm}^{-1}$ and $468-471 \mathrm{~cm}^{-1}$, respectively. The band at $968 \mathrm{~cm}^{-1}$ was assigned to a $\mathrm{Si}-\mathrm{OH}$ stretching vibration [10]. The decrease in peak intensity of the band at $\sim 780$ $\mathrm{cm}^{-1}$ in $\mathrm{RH}-x \mathrm{In}$ was due to the formation of $\mathrm{Si}-\mathrm{O}-\mathrm{In}$ [8]. RH-20In has the lowest intensity because of the high percentage of indium metal incorporated into RH.

\subsubsection{SEM/EDX and AAS analysis}

Figure 2 shows the SEM images of RH-blank and RH- $x$ In catalysts. These catalysts were highly porous. The porosity gradually increased from RH-5In to RH-20In, as the percentage of incorporated indium increased. Different sized spherical particles were observed in the RH-5In, RH-10In,
RH-15In, and RH-20In.

The EDX and AAS analysis (Table 1) shows the presence of $\mathrm{In}^{3+}$ ions in all the RH-xIn catalysts. From the EDX results, the percentage of indium incorporated increased from $3.29 \%$ to $6.60 \%$ for catalysts RH-5In and RH-20In, respectively. EDX is a surface analysis technique, while AAS is used to determine the bulk ion concentration. Therefore, it is not surprising the EDX values are much lower than the AAS values. From the AAS measurements $\mathrm{In}^{3+}$ ions increased from $5.6 \%$ to $11.2 \%$ for catalysts RH-5In and RH-20In, respectively.

Table 1 Elemental composition of the catalysts determined by EDX and AAS

\begin{tabular}{lccccc}
\hline \multirow{2}{*}{ Sample } & \multicolumn{3}{c}{$\begin{array}{c}\text { Element content determined by EDX } \\
(\%)\end{array}$} & $\begin{array}{c}\text { In content } \\
\text { determined by } \\
\end{array}$ & \multicolumn{5}{c}{$\mathrm{Si}$} & $\mathrm{C}$ & $\mathrm{O}$ & $\mathrm{In}$ & AAS (\%) \\
\hline RH-blank & 33.73 & 9.27 & 57.00 & - & - \\
RH-5In & 25.45 & 8.05 & 63.02 & 3.29 & 5.6 \\
RH-10In & 36.35 & 9.79 & 49.04 & 4.82 & 8.6 \\
RH-15In & 29.45 & 9.87 & 54.23 & 6.44 & 10.2 \\
RH-20In & 20.90 & 5.09 & 63.02 & 6.60 & 11.2 \\
\hline
\end{tabular}

\subsubsection{TEM analysis}

Figure 3 shows the TEM micrographs of the prepared samples. There were two types of morphologies: worm-like mesostructure and spherical particles. The TEM of RH-blank (Fig. 3(a)) showed a worm-like porous network structure [26]. The indium incorporated catalysts consisted of non-uniform spherical particles. In general, the size of the particles increased as the amount of indium incorporation increased.
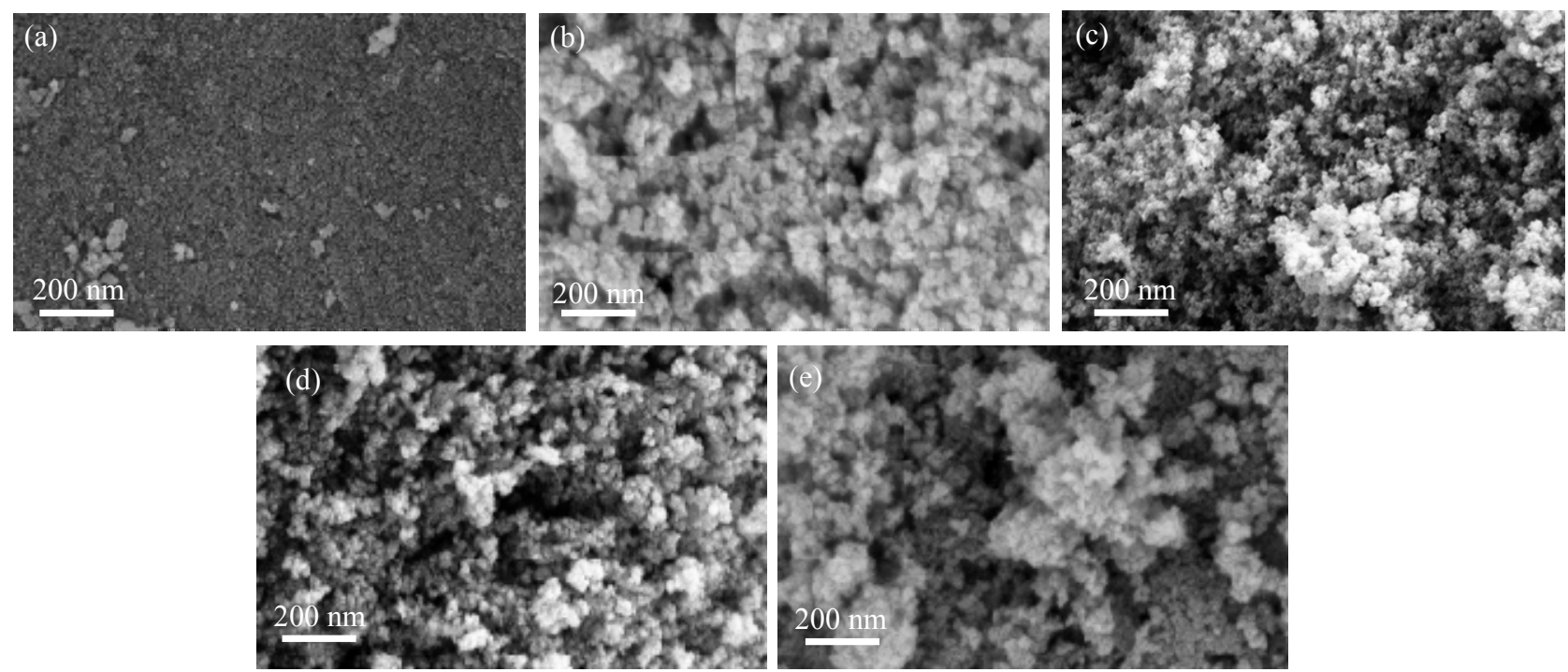

Fig. 2. SEM images of RH-blank (a), RH-5In (b), RH-10In (c), RH-15In (d), and RH-20In (e). 

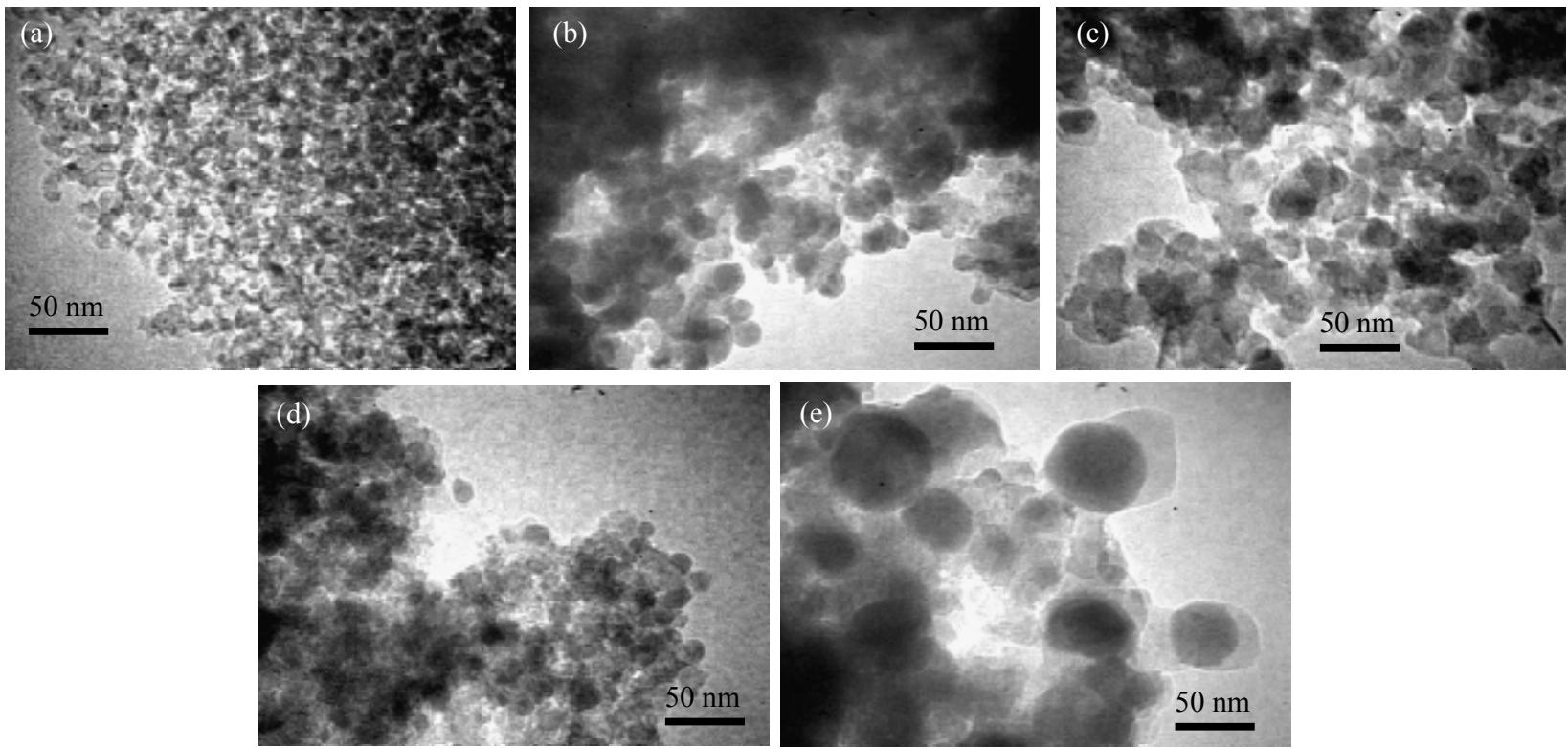

Fig. 3. TEM images of RH-blank (a), RH-5In (b), RH-10In (c), RH-15In (d), and RH-20In (e).

\subsubsection{XRD analysis}

Figure 4 shows the high-angle XRD analysis of all the samples. All samples were amorphous as shown by the broad band around $2 \theta=25^{\circ}$. The intensity of the peak decreased from RH-blank to RH-20In due to the incorporation of indium metal in silica. Crystalline peaks were not detected for the samples.

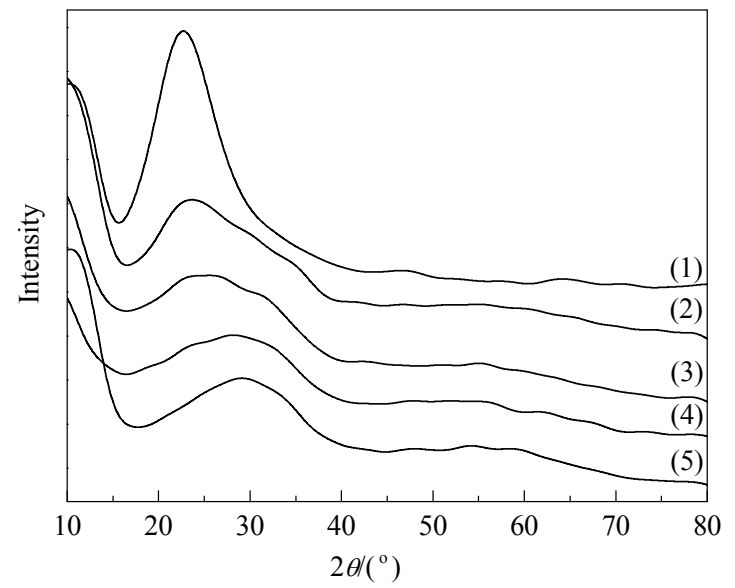

Fig. 4. XRD patterns for RH-blank (1), RH-5In (2), RH-10In (3), RH-15In (4), and RH-20In (5).

\subsection{5 $\quad \mathrm{N}_{2}$ adsorption-desorption analysis}

The $\mathrm{N}_{2}$ adsorption-desorption isotherms are shown in Fig. 5. All the samples exhibit type IV isotherms. The differences in textural properties results in two types of hysteresis loops: $\mathrm{H} 1$ for RH-blank and $\mathrm{H} 3$ for the indium incorporated

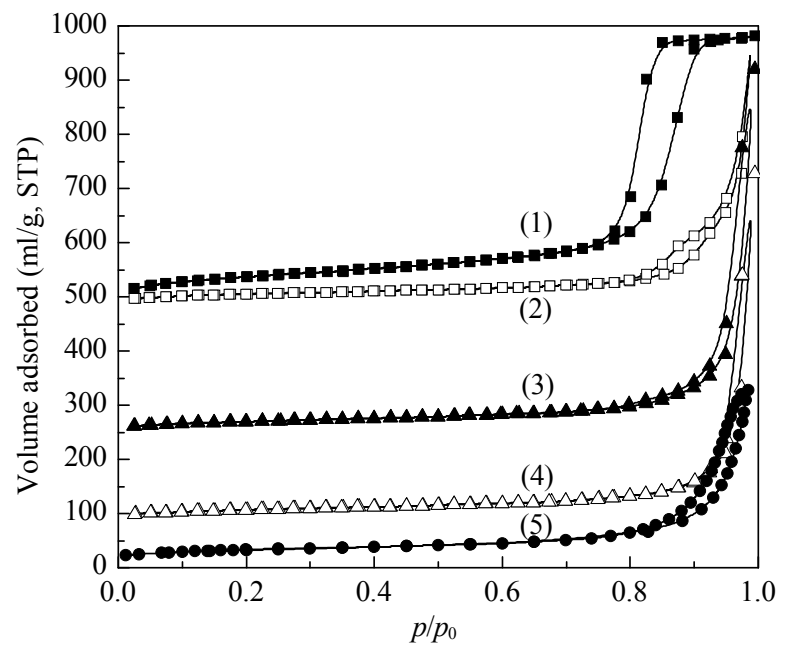

Fig. 5. $\mathrm{N}_{2}$ adsorption-desorption of RH-blank (1), RH-5In (2), RH-10In (3), RH-15In (4), and RH-20In (5).

RH-xIn. The H1 hysteresis loop in the range of relative pressure $0.8<p / p_{0}<0.9$ of RH-blank is characteristic of porous material consisting of uniform particles with small pore distributions [10]. Whereas the catalysts RH-xIn have H3 hysteresis loops representing a wide distribution of pore sizes [27]. It was interesting to observe that the pore size increased as the metal loading increased. RH-blank recorded the smallest pore size range of 4.10-7.70 nm while RH-20In showed the largest pore size range from $26.50-31.50 \mathrm{~nm}$. The pore volume, specific surface area, and range of pore size for all the samples are shown in Table 2. The indium loading in silica caused the surface area of 
Table $2 \quad \mathrm{~N}_{2}$ adsorption-desorption analyses of RH-blank and RH-xIn

\begin{tabular}{lccc}
\hline Sample & $\begin{array}{c}\text { Pore size } \\
(\mathrm{nm})\end{array}$ & $\begin{array}{c}\text { Pore volume } \\
(\mathrm{ml} / \mathrm{g})\end{array}$ & $\begin{array}{c}\text { BET surface area } \\
\left(\mathrm{m}^{2} / \mathrm{g}\right)\end{array}$ \\
\hline RH-blank & $4.1-7.7$ & 0.9 & 206.1 \\
RH-5In & $4.5-9.3$ & 0.9 & 76.4 \\
RH-10In & $18.0-21.0,22.0-28.0$ & 1.1 & 86.1 \\
RH-15In & $20.9-30.2$ & 1.0 & 77.7 \\
RH-20In & $26.5-31.5$ & 0.5 & 96.7 \\
\hline
\end{tabular}

RH-blank to decrease from 206 to $\sim 85 \mathrm{~m}^{2} / \mathrm{g}$ (RH-xIn) because the indium particles blocked the smaller pores [8].

\subsection{Benzoylation of toluene}

\subsubsection{Effect of temperature}

The benzoylation of toluene was studied in the temperature range 363 to $383 \mathrm{~K}$ using RH-10In catalyst and the results are shown in Fig. 6. The major products are 4-methylbenzophenone (4-MBP) and 2-methylbenzophenone (2-MBP) while, the minor product is 3-methylbenzophenone (3-MBP). At $363 \mathrm{~K}$, the conversion was $76 \%$ and it increased to $100 \%$ at $373 \mathrm{~K}$. Further increases in temperature did not affect the percentage conversion. The selectivity for 4-MBP increased from $75 \%$ to $78 \%$ as the temperature increased from 363 to $373 \mathrm{~K}$, then decreased to $57 \%$ at $383 \mathrm{~K}$. The selectivity for 3-MBP increased from 0 to $18 \%$ as the temperature increased from 363 to $383 \mathrm{~K}$. It is interesting to note that the 3-MBP selectivity increased as the reaction temperature increased. This might be due to the fact that the 3-MBP is a thermodynamically stable product [28]. It should be noted that there was no product formation when the reaction was carried out at room temperature.

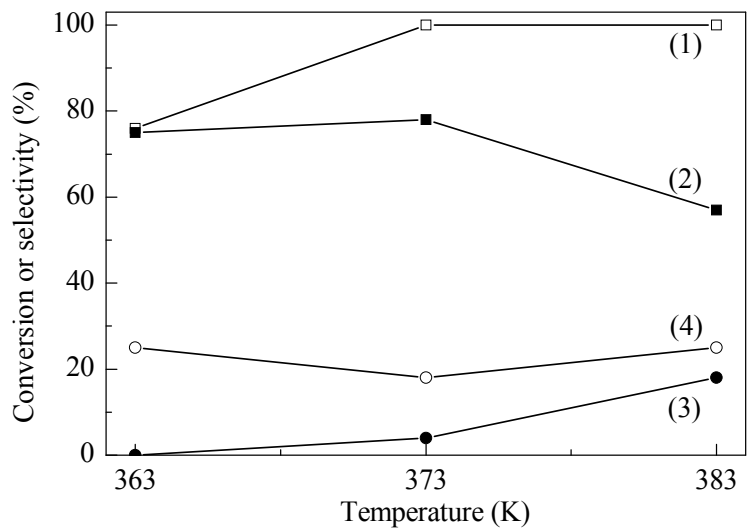

Fig. 6. Effect of temperature on the conversion of benzoyl chloride (1) and selectivity for 4-MBP (2), 3-MBP (3), and 2-MBP (4). Reaction conditions: toluene to benzoyl chloride molar ratio $15: 1$, RH-10In $0.10 \mathrm{~g}, 1 \mathrm{~h}$

\subsubsection{Effect of reaction time}

Figure 7 shows the effect of reaction time for benzoylation of toluene carried out over a period of $3 \mathrm{~h}$. The reaction resulted in $65 \%$ conversion at $0.5 \mathrm{~h}$ and increased to $100 \%$ conversion at $1 \mathrm{~h}$. The selectivity for the para-product (4-MBP) decreased as the time increased. It is known that in non-polar solvents (in this case toluene), the ortho-product is favored [29]. However, in our study, it is interesting to observe that the selectivity for 2-MBP increased with time.

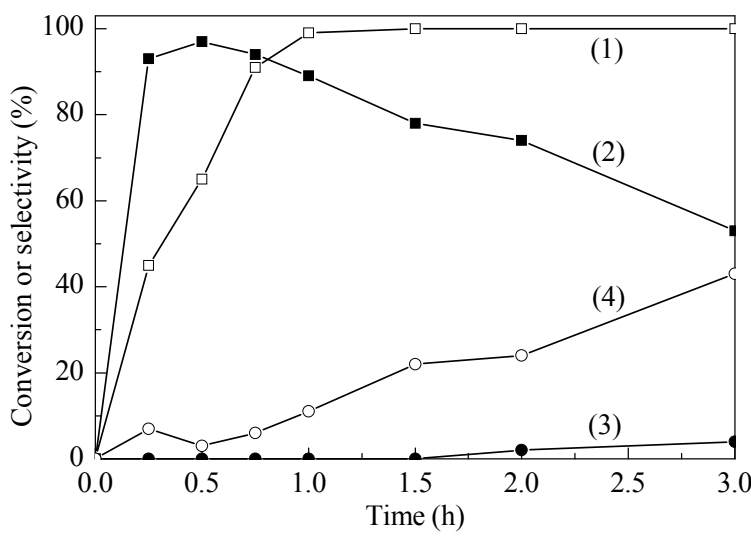

Fig. 7. Effect of time on the conversion of benzoyl chloride (1) and selectivity for 4-MBP (2), 3-MBP (3), and 2-MBP (4). Reaction conditions: $373 \mathrm{~K}$, toluene to benzoyl chloride molar ratio 15:1, RH-10In $0.10 \mathrm{~g}$.

\subsubsection{Effect of molar ratio of reactants}

Figure 8 shows the conversion and selectivity for MBP's at different molar ratios of toluene to benzoyl chloride over a $1 \mathrm{~h}$ reaction time. The molar ratios of 15:1 and 20:1 achieved $100 \%$ conversion, while for molar ratios of $5: 1$ and $10: 1,94 \%$ and $93 \%$ conversions were obtained, respec-

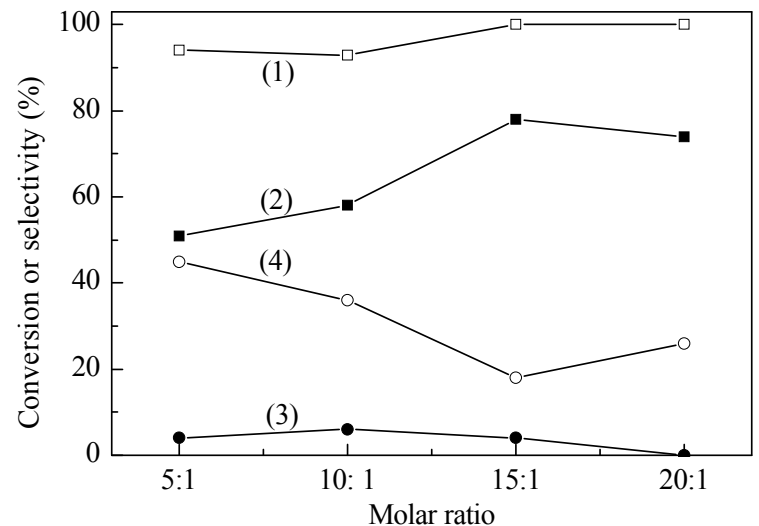

Fig. 8. Effect of reactant molar ratio of toluene to benzoyl chloride on the conversion of benzoyl chloride (1) and selectivity for 4-MBP (2), 3-MBP (3), and 2-MBP (4). Reaction conditions: $373 \mathrm{~K}, \mathrm{RH}-10 \mathrm{In}$ $0.10 \mathrm{~g}, 1 \mathrm{~h}$. 
tively. The change in molar ratio influenced the selectivity for the three products. The selectivity for 4-MBP increased (51\% to $78 \%$ ), but the selectivity for 2-MBP decreased $(45 \%$ to $18 \%)$ as the concentration of toluene increased from $5: 1$ to $15: 1$. The increase in concentration of toluene minimized the formation of 3-MBP, as the selectivity for 3-MBP decreased with increasing concentration of toluene.

\subsubsection{Effect of catalyst amount}

The effect of catalyst amount on the benzoyl chloride conversion and selectivity for MBP was studied in the mass range of 0.05 to $0.20 \mathrm{~g}$ and the results are represented in Fig. 9. The conversion increased from $85 \%$ to $100 \%$ when the catalyst mass increased from 0.05 to $0.10 \mathrm{~g}$ and was maintained at $100 \%$ when the mass was $0.20 \mathrm{~g}$. The slight variations in the selectivity for 4-MBP and 2-MBP may be just due to experimental error.

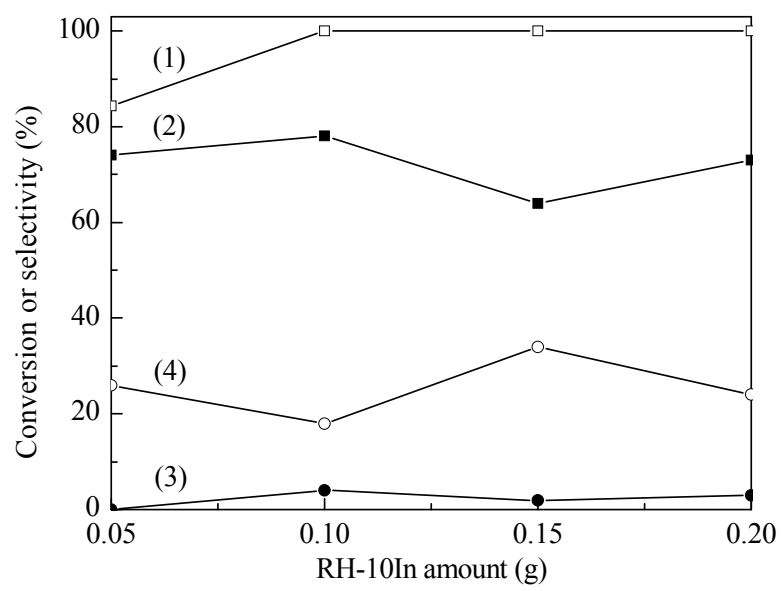

Fig. 9. Effect of RH-10In amount on the conversion of benzoyl chloride (1) and selectivity for 4-MBP (2), 3-MBP (3), and 2-MBP (4). Reaction conditions: $373 \mathrm{~K}$, toluene to benzoyl chloride molar ratio $15: 1,1 \mathrm{~h}$.

\subsubsection{Effect of indium loading}

The catalytic activities of the different metal loaded catalysts RH- $x$ In are shown in Fig. 10. RH-5In showed the lowest conversion of $65 \%$, while others gave $100 \%$ conversion. It should be noted that 3-MBP was only detected in the presence of RH-20In catalyst. The selectivity of 4-MBP showed a decreasing trend from $\mathrm{RH}-5 \mathrm{In}(97 \%)$ to $\mathrm{RH}-15 \mathrm{In}$ (69\%), and then showed a slight increase to $78 \%$ for RH-20In. No products were detected when RH-blank was used. This shows that the indium ion is the catalytic active species. In our study, RH-10In gave $100 \%$ conversion and $89 \%$ selectivity for 4-MBP. For the same reaction, Choundhary et al. [19] reported that for a $20 \% \quad \mathrm{In}_{2} \mathrm{O}_{3}$ loaded/Si-MCM-41 catalyst a conversion of $85 \%$ at $383 \mathrm{~K}$

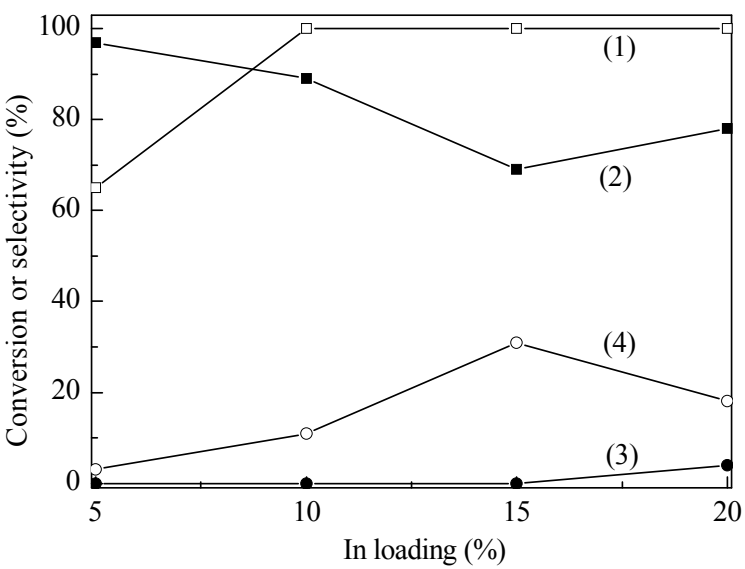

Fig. 10. Effect of In loading on the conversion of benzoyl chloride (1 and selectivity for 4-MBP (2), 3-MBP (3), and 2-MBP (4). Reaction conditions: catalyst $0.10 \mathrm{~g}, 373 \mathrm{~K}$, toluene to benzoyl chloride molar ratio $15: 1,1 \mathrm{~h}$.

in $2.5 \mathrm{~h}$ was achieved. In comparison, the catalyst prepared in this study can be used at lower temperature, lower metal loading, shorter reaction times and gives higher conversion percentage yields.

\subsubsection{Leaching and reusability of $\mathrm{RH}-10 \mathrm{In}$ in benzoylation of toluene}

The reusability of the catalyst was tested under the optimum conditions and the results are shown in Table 3 . The catalysts can be used up to three times and the conversion at the 3 rd reuse slightly decreased to $94 \%$. There were some differences in the product selectivity.

Table 3 Reusability of RH-10In

\begin{tabular}{lcccc}
\hline \multirow{2}{*}{ Catalyst } & $\begin{array}{c}\text { Conversion } \\
(\%)\end{array}$ & \multicolumn{3}{c}{ Selectivity (\%) } \\
\cline { 3 - 5 } & 100 & 86 & 14 & 0 \\
\hline Fresh & 100 & 79 & 21 & 0 \\
1st-reuse & 100 & 80 & 16 & 4 \\
2nd-reuse & 94 & 53 & 39 & 8 \\
3rd-reuse & &
\end{tabular}

Reaction conditions: catalyst $0.10 \mathrm{~g}, 373 \mathrm{~K}$, toluene to benzoyl chloride molar ratio $20: 1,1 \mathrm{~h}$.

The leaching test was performed to ensure catalyst heterogeneity. The reaction was allowed to proceed for $0.5 \mathrm{~h}$, before the catalyst was removed and then the filtered solution was refluxed (without catalyst) for $2 \mathrm{~h}$. The results are shown in Fig. 11. The conversion increased slightly from $47 \%$ at $0.5 \mathrm{~h}$ to $50 \%$ at $2 \mathrm{~h}$ after catalyst removal. The slight increase in conversion is insignificant and maybe due to solvent loss over longer reaction times. It can be safely concluded that the leaching effects are negligible for RH-10In and its catalytic activity is heterogeneous. 


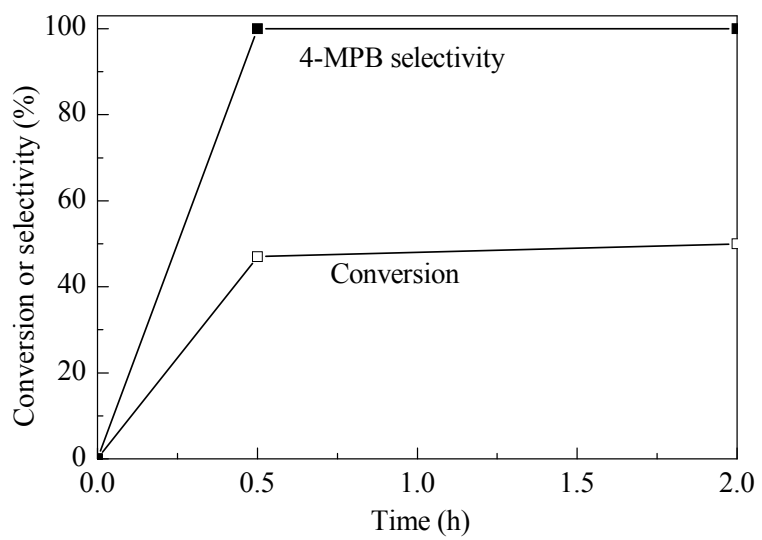

Fig. 11. Effect of catalyst removal and leaching on RH-10In. Catalyst was removed after $0.5 \mathrm{~h}$.

\section{Conclusions}

Indium incorporated silica catalysts with different indium loadings were successfully prepared via a simple, economical, and environmental friendly sol-gel method. The synthesis was carried out at room temperature and the resulting catalyst did not require calcination. The $\mathrm{N}_{2}$ adsorption-desorption and XRD analyses showed that these catalysts were mesoporous. Increasing indium metal loading increased the pore size of the catalyst. The selectivity for 4-MBP increased as the concentration of the toluene increased. The selectivity for 4-MBP was always higher than both 2-MBP and 3-MBP with all the catalysts studied. $\mathrm{RH}-10 \mathrm{In}$ showed the highest selectivity for 4-MBP under the optimum conditions.

\section{References}

1 Liou T H, Yang C C. Mater Sci Eng B, 2011, 176: 521

2 Kim K D, Kim H T. J Sol-Gel Sci Technol, 2002, 25: 183

3 Markowitz M A, Schoen P E, Kust P, Gaber B P. Colloid Surface A, 1999, 150: 85

4 Nassar E J, Nassor E C De O, Ávila L R, Pereira P F S, Cestari A, Luz L M, Ciuffi K J, Calefi P S. J Sol-Gel Sci Technol, 2007, 43: 21

5 Baccile N, Babonneau F, Thomas B. J Mater Chem, 2009, 19: 8537
6 Moleski R, Leontidis E, Krumeich F. J Colloid Interface Sci, 2006, 302: 246

7 Chiarakorn S, Areerob T, Grisdanurak N. Sci Tech Adv Mater, 2007, 8: 110

8 Ahmed A E, Adam F. Microporous Mesoporous Mater, 2007, 103: 284

9 Amutha K, Ravibaskar R, Sivakumar G. Int J Technol Appl, 2010, 4: 61

10 Adam F, Chew T S, Andas J. J Sol-Gel Sci Technol, 2011, 59: 580

11 Dimitriev Y, Ivanova Y, Iordanova R. J Univ Chem Technol Metallurgy, 2008, 43: 181

12 Singh A P, Bhattacharya D, Sharma S. J Mol Catal A, 1995 , 102: 139

13 Gawande M B, Deshpande S S, Sonavane S U, Jayaram R V. J Mol Catal A, 2005, 241: 151

14 Laidlawa P, Bethell P D, Brown S M, Hutchings G J. J Mol Catal A 2001, 174: 187

15 Arata K, Nakamura H, Shouji M. Appl Catal A, 2000, 197: 213

16 Choudhary V R, Jana S K, Narkhede V S. Appl Catal A, 2002, 235: 207

17 Nguyen L T L, Le K K A, Phan N T S. Chin J Catal, 2012, 33: 688

18 Bordoloi A, Mathew N T, Devassy B M, Mirajkar S P, Halligudi S B. J Mol Catal A, 2006, 247: 58

19 Liu E, Locke A J, Frost R L, Martens W N. J Mol Catal A, 2012, 353-354: 95

20 Choudhary V R, Jha R. Appl Catal A, 2007, 333: 42

21 Choudhary V R, Jana S K. J Mol Catal A, 2002, 184: 247

22 Jang D O, Moon K S, Choa D H, Kim J S. Tetrahedron Lett, 2006, 47: 6063

23 Wildermann A, Foricher Y, Netscher Y T, Bonrath W. Pure Appl Chem, 2007, 79, 11: 1839

24 Miyai T, Onishi Y, Baba A. Tetrahedron, 1999, 55: 1017

25 Miyai T, Onishi Y, Baba A. Tetrahedron Lett, 1998, 39: 6291

26 Xia Y, Dai H, Jiang H, Zhang L, Deng J, Liu Y. J Hazard Mater, 2011, 186: 84

27 Chang S S, Clair B, Ruelle J, Beauchéne J, FRenzo F D, Quignard F, Zhao G J, Yamamoto H, Gril J. J Exp Bot, 2009, 60, 11: 3023

28 Vinu A, Krithiga T, Gokulakrishnan N, Srinivasu P, Anandan S, Ariga K, Murugesan V, Balasubramanian V V, Mori T. Microporous Mesoporous Mater, 2007, 100: 87

29 Waghmare A A, Bose P, Pati H N. Pharma Chem, 2010, 2: 212 